\title{
D'un « déblayage » à l'autre : renouveaux scéniques à Bayreuth de 1930 à 1970
}

\section{Helga Finter}

\section{(2) OpenEdition}

1 Journals

Édition électronique

URL : https://journals.openedition.org/rbnu/2328

DOI : 10.4000/rbnu.2328

ISSN : 2679-6104

Éditeur

Bibliothèque nationale et universitaire de Strasbourg

\section{Édition imprimée}

Date de publication : 1 mai 2013

Pagination : 38-49

ISBN : 9782859230432

ISSN : 2109-2761

\section{Référence électronique}

Helga Finter, « D'un « déblayage » à l'autre : renouveaux scéniques à Bayreuth de 1930 à 1970 », La Revue de la BNU [En ligne], 7 | 2013, mis en ligne le 01 mai 2013, consulté le 08 août 2021. URL : http:// journals.openedition.org/rbnu/2328; DOI : https://doi.org/10.4000/rbnu.2328

\section{(c) (i) (2) (2)}

La Revue de la BNU est mise à disposition selon les termes de la Licence Creative Commons Attribution - Pas d'Utilisation Commerciale - Partage dans les Mêmes Conditions 4.0 International. 
MAQUETTES DES DECORS DE LA CREATION DE *PARSIFAL *

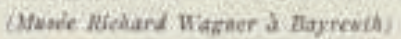

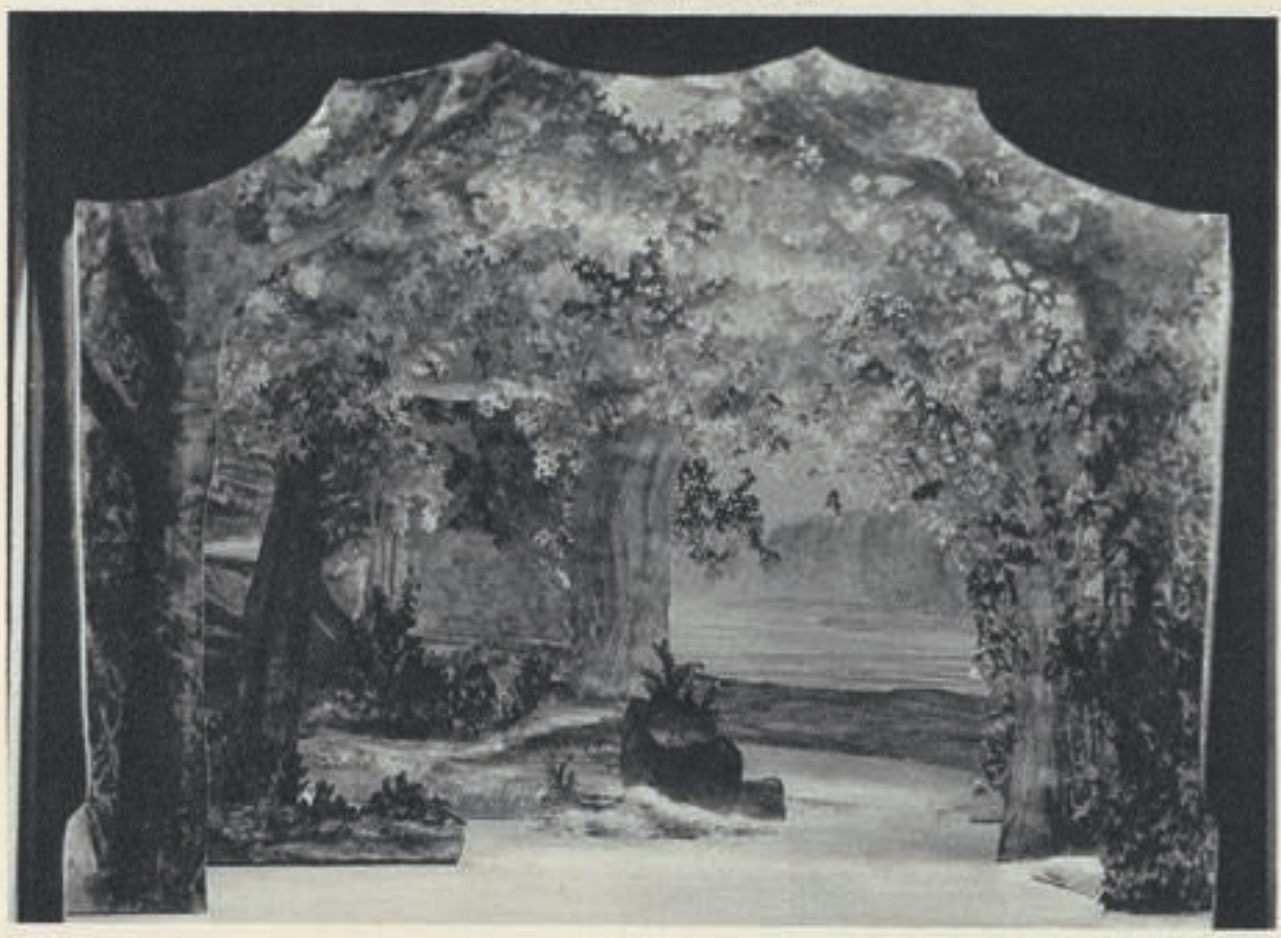

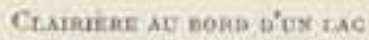

Shaquette de soukowisy.

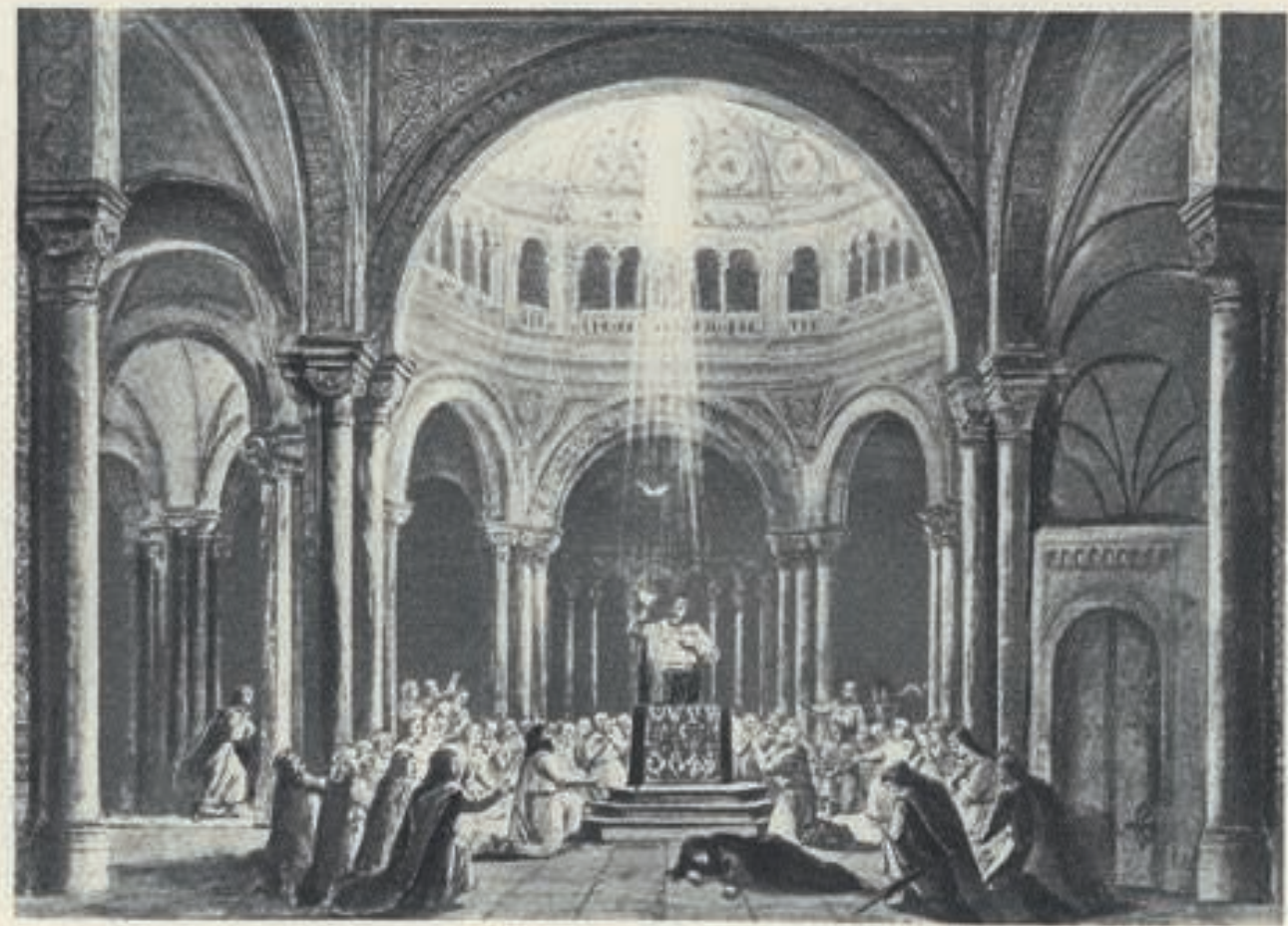

L. Traphe at Geus.

Maquette de Joukamaky. 


\section{D'un «déblayage» à l'autre : \\ renouveaux scéniques à Bayreuth de I930 à I970}

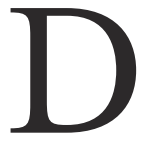

ans Parsifal, seule œuvre expressément écrite par Wagner pour le nouveau théâtre du festival, selon les indications scéniques de la dernière partie du premier acte, le héros, guidé par Gurnemanz, entre dans le château du Graal - le Gralsburg. Il traverse des " couloirs ascendants, bordés de murs " avant de pénétrer dans " une salle sompteuse ", " un hall dont les colonnes soutiennent une coupole qui couvre la salle d'un réfectoire " ${ }^{1}$. Le projet scénique du peintre Paul von Joukowsky pour la première représentation à Bayreuth, le 28 juillet 1882, fut réalisé par Max Brückner. Il montre une toile de fond richement colorée qui rappelle le style byzantin des cathédrales avec un intérieur à coupoles, comme Saint Marc à Venise, Sainte Sophie à Constantinople ou Santa Maria Assunta de Sienne. Ce lieu scénique, s'ouvrant vers l'infini bleu des cintres, impressionne par sa richesse de couleurs moirées d'ors. Utilisée, comme les décors des autres actes, pour toutes les mises en scène de Parsifal à Bayreuth, de 1882 jusqu'en 1931, cette image scénique est quasiment devenue l'emblème du lieu sacré abritant le Graal.

La mise en scène de l'œuvre par Stefan Herheim, donnée de 2008 à 2012 à Bayreuth, le rappelle quand elle transforme l'espace du premier acte en une citation de ce décor originel. Auparavant dans ce même acte, Herheim introduit déjà, à partir de tout un montage, d'autres citations de lieux : dans le fond de scène, la façade arrière de la villa Wahnfried s'ouvre vers un intérieur rappelant la salle en rotonde de la maison de Wagner tandis que le milieu de l'avant-scène est bordé par la tombe du compositeur. L'ouverture en hauteur du nouvel espace, grâce à une coupole immense, submerge le spectateur contemporain : il retrouve ainsi l'expé- rience d'une vision qui, portée par le motif musical du Graal, éveille en lui le désir d'une rédemption.

Cette mise en scène d'Herheim thématise sur plusieurs niveaux de lecture - psychologique, biographique, historique - le contexte de la production et de la réception de l'œuvre dans une esthétique rappelant celle du cinéaste Hans Jürgen Syberberg. L'image de la salle du Graal s'y avère être l'emblème, dans la scénographie wagnérienne, du mythe d'une rédemption. On la retrouve en effet au second acte, qui se tient dans le royaume de Klingsor, quand Parsifal évoque le Graal devant Kundry ; mais la coupole disparaît quand l'aigle allemand avec une croix gammée en descend pour surmonter la salle à présent fermée en hauteur : elle est à nouveau transformée et devient avec ses drapeaux rouges nazis le lieu d'un rite politique. Au troisième acte, c'est la citation d'une autre salle avec une autre coupole qui métamorphose la salle du Graal : celle du parlement de Berlin et de la coupole en verre de Norman Foster pour l'ancien Reichstag. Cette coupole reflète en miroir l'orchestre invisible avec son chef et les spectateurs dans la salle. Le symbole d'une rédemption promise devient alors dans cette lecture actuelle celui d'une religion de l'art, dont le seul support ne peut être aujourd'hui que le désir du spectateur renvoyé à luimême ${ }^{2}$.

Beaucoup de critiques d'obédiences politiques différentes ont fêté cette mise en scène comme l'annonce d'un second renouveau de Bayreuth, comme la " rédemption " historique et politique de l'œuvre de Wagner pour une nouvelle génération ${ }^{3}$. Car avec le travail d'Herheim, l'évacuation de la politique et de ses relents nazis, que Wieland Wagner avait initiée avec le Parsifal de 1951 (il annonçait lui aussi, avec la suppression de la 
coupole du Graal, un " Neu-Bayreuth " - un nouveau Bayreuth), sembla alors parachevée 4 .

Une telle suppression avait pourtant déjà fait grand bruit en 1934, quand Winifred Wagner fit représenter une salle du Graal sans coupole par le décorateur Alfred Roller, engagé à l'instigation d'Adolf Hitler modification qui fut d'ailleurs abandonnée dès 1936 quand Wieland Wagner restitua comme décorateur cet élément emblématique du Graal à Bayreuth ${ }^{5}$ et qu'il le reprit dans sa propre scénographie de l'œuvre, en $1937^{6}$.

L'exemple de ce nouveau " déblayage » de la scène de Bayreuth nous tient lieu de point de départ pour nous interroger sur l'esthétique des mises en scène à Bayreuth entre 1930 et 1970. Avant de l'aborder, quelques mots seront nécessaires concernant la conception et la place de la mise en scène selon Richard Wagner lui-même.

\section{Wagner homme de théâtre}

Wagner considérait que l'opéra de son temps était devenu obsolète, à la fois sur les plans esthétique, éthique et social. Avec le " Wort-Ton-Drama " (drame musical) qu'il concevait comme "Gesamtkunstwerk " (œuvre d'art totale), l'écrivain, compositeur, chef d'orchestre, metteur en scène et penseur proposa une profonde réforme de l'institution opératique, visant à abolir les frontières avec les autres arts scéniques pour leur rendre l'impact politique, social et psychologique qu'ils devaient avoir sur le spectateur et la société.

A partir de l'idée du festival (Festspiel), empruntée aux Grecs anciens, un lieu et un temps furent fixés pour un théâtre qui, selon le modèle de la tragédie grecque, devait restituer avec tous les arts de la scène à la fois l'unité de l'homme et celle de la société. Cette visée psycho-politique cherchait à réduire les fractures, à combler les manques du sujet et de la société allemandes de son temps, et à surmonter leur détresse ("Not ") grâce à la puissance du mythe, que le drame musical devait projeter en vue d'une unité, à la fois psychique et nationale, à conquérir. La forme du " Gesamtkunstwerk " modelait une telle unité par une synthèse des arts ; elle abolissait la séparation en disciplines indépendantes, qui était intervenue à la fin $\mathrm{du} 17^{\mathrm{e}}$ siècle français, et permettait ainsi, par la résurrection du mythe, une communion avec l'inconscient du spectateur.

Les arts de l'écoute - la poésie, le chant, la musique et les arts du regard - le geste et la mimique des chanteurs, la danse, la peinture - devaient fusionner dans une œuvre d'art totale, censée abolir leurs spécificités respectives au profit d'une totalité, et ce de la même façon que le spectateur était appelé à transcender son désir singulier au profit d'une unité sociale et nationale, en le fixant dans le mythe rassembleur ${ }^{7}$.

Le théâtre érigé à Bayreuth fournissait, en tant que machine de perception et de représentation, la condition matérielle d'une telle fusion psychophysique. Le fait de cacher la fosse d'orchestre créait une acoustique spécifique qui enveloppait salle et scène dans un même espace sonore, tandis que la disposition des sièges en amphithéâtre, associée à la création d'un second proscénium, rendait la scène, pourvue de cintres et de trappes, à la fois proche et lointaine, comme née d'un rêve ${ }^{8}$.

En s'attelant avec ses collaborateurs à la mise en scène, Wagner n'avait pu mettre au point à Bayreuth qu'une seule mise en scène le satisfaisant - celle du Parsifal de 1882, réservée expressément au nouveau théâtre. Le Ring de l'inauguration de la nouvelle maison en 1876 n'avait que partiellement correspondu à ses attentes, comme d'ailleurs les mises en scène antérieures. Cosima, dans son Journal, nous rapporte même un propos selon lequel il souhaitait « après avoir inventé l'orchestre invisible, inventer le théâtre invisible " 9 .

L'aspect visuel du spectacle et son articulation avec le chant et la musique posaient problème : les mimiques, les mouvements et gestes des chanteurs, leurs masques et leurs costumes ainsi que le décor devaient être repensés. Tributaires de la rhétorique opératique d'une part, de l'esthétique historicisante d'autre part, tout en contribuant pourtant à son succès ${ }^{10}$, ils restaient encore trop marqués par le théâtre de l'époque et empêchaient la fusion audiovisuelle que le compositeur espérait offrir à son nouveau public. Car le jeu, les costumes, les masques comme le décor, n'étaient en fait plus conçus par Wagner comme simples effets de réel, comme illustrations ou coulisses ; ils étaient pour lui des symboles ou des actants symboliques, changeant et se transformant insensiblement comme les états d'âme des personnages, suggérés déjà par la musique. Celle-ci, caractérisant les personnages autant que l'aspect visuel, se manifestait par des sources sonores différentes : à côté du chant, le tissu des leitmotive provenant de l'orchestre anticipait, réfléchissait ou rappelait pour les personnages les motivations inconscientes relevant du mythe et du destin, et pouvait même aussi contredire les paroles énoncées par le chant. 


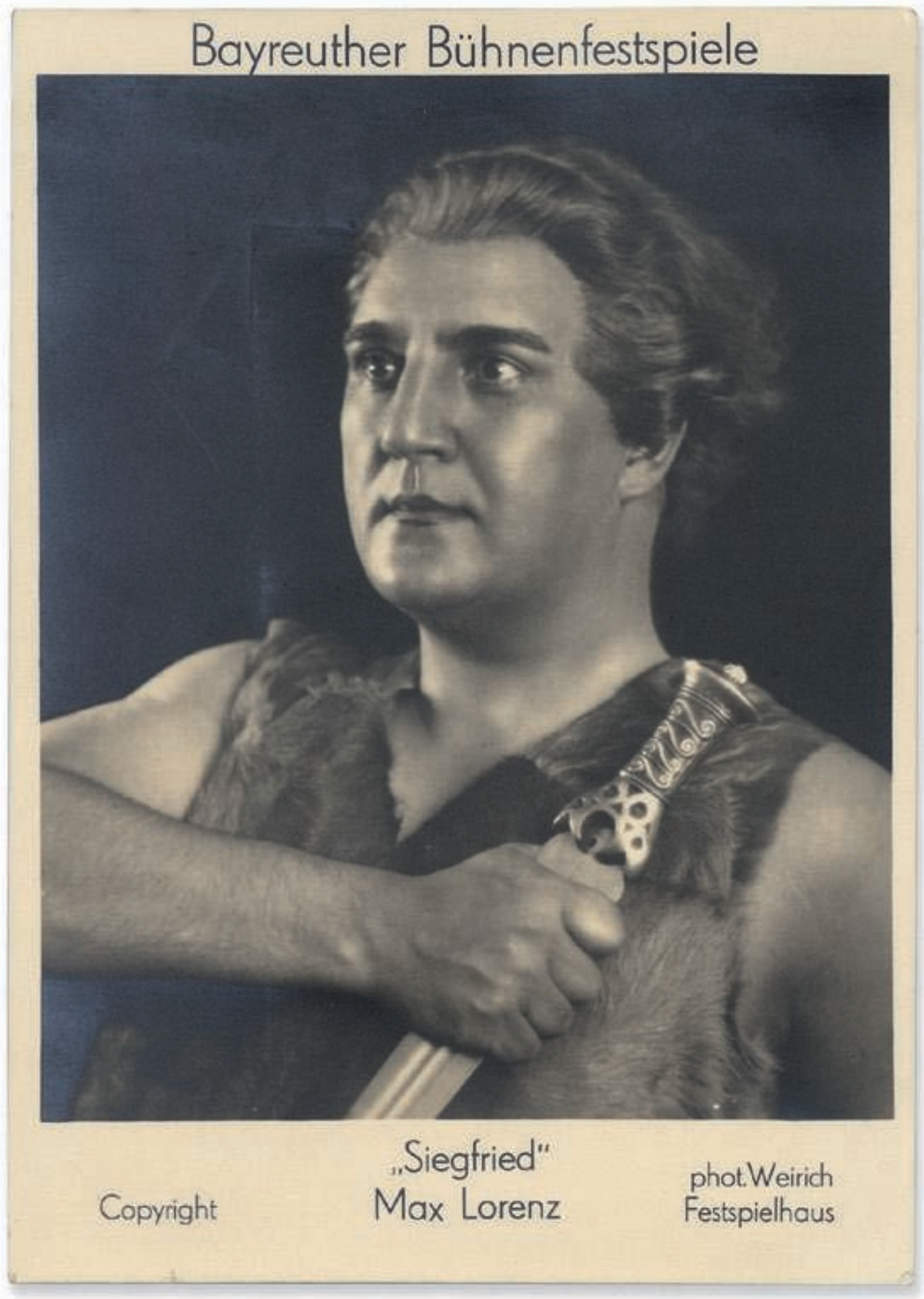

Le ténor Max Lorenz dans Siegfried, carte postale éditée lors de la représentation du Festival de Bayreuth de 1937 (direction musicale W. Furtwängler, mise en scène H. Tietjen, décors E. Preetorius ; coll. BNU) 
Cosima, en consolidant le répertoire à Bayreuth, ne suivit cependant pas entièrement les vœux de Wagner : elle reconstitua fidèlement la mise en scène de Parsifal qu'il avait approuvée, mais conserva des éléments scéniques du Ring tenus seulement pour passables par Wagner, ainsi que des productions antérieures à Bayreuth. Avec l'aide des frères Brückner qui en avaient créé les décors et costumes, elle fixa une esthétique visuelle tributaire du style historicisant d'un MoyenÂge imaginaire, chère à l'époque wilhelminienne, et qui avait déjà fait le succès des Maîtres chanteurs ou de Tristan des décennies auparavant. Pour le jeu et le chant, elle œuvra cependant dans l'esprit de Wagner en imposant le "Sprechgesang " et en éliminant des tics opératiques, comme le tremolo ou les gestes superflus ${ }^{11}$.

Jusqu'en 1910 régna ainsi, à Bayreuth comme en dehors, une uniformité des costumes et décors wagnériens, favorisée d'une part par une production iconographique dont pouvaient s'inspirer d'autres théâtres, d'autre part par le commerce de l'atelier Brückner à Cobourg qui vendait des copies des décors de Bayreuth aux théâtres du monde entier ${ }^{12}$.

\section{Réformes hors de Bayreuth jusqu'en 1930}

Les projets de réforme de la mise en scène wagnérienne proposés dès 1887 par des théoriciens comme Adolphe Appia ou plus tard Gordon Craig, par des artistes comme Meyerhold ou Tatlin, ou par les artistes de l'époque de la République de Weimar, comme par exemple Jürgen Fehling et Karl Dülberg pour le Vaisseau fantôme à la Krolloper de Berlin en 1929, n'eurent pas d'impact sur la pratique scénique de Bayreuth. Ces réformes concernaient d'abord le décor fixe, les costumes, et la chorégraphie des gestes et mouvements. Elles cherchaient à vider la scène de la peinture historicisante, des accessoires et costumes kitsch, pour les remplacer par un espace ouvert et mobile, dessiné par la lumière, afin de visualiser, selon Appia, les affects suggérés par la musique. Une sobriété abstraite des costumes et la chorégraphie de masse des chœurs, ainsi qu'une gestuelle motivée par la musique devaient, de même, y contribuer.

Siegfried Wagner, attelé à la difficile consolidation financière du festival, releva le défi d'intégrer, comme dans un patchwork, de nouveaux éléments de décor et de costumes à ceux qui, vieillis, étaient devenus inopérants. Il ne livra une lecture neuve que pour une seule œuvre, avec sa dernière production du Tannhäuser de 1930, dont il ne put connaître le succès de son vivant. Une régie subtile de la lumière, soutenue par des projections, caractérisait déjà son Ring grâce à une nouvelle installation d'éclairage, acquise en 1928, ainsi qu'à des décors mobiles plus sobres, aux couleurs inspirées par les expressionnistes. De même ${ }^{13}$, une chorégraphie contemporaine de Rudolf von Laban pour le premier acte de ce Tannhäuser contribuait à cette timide modernisation. Une première réforme systématique fut cependant entreprise seulement après sa mort, par son épouse Winifred qui lui succéda.

\section{Un premier « déblayage » sous la croix gammée}

L'amie intime d'Hitler, mécène et soutien du parti nazi de la première heure, réussit, grâce à son lien personnel avec le Führer, l'exploit de garantir le financement du festival par Hitler et l'Etat nazi, tout en gardant à Bayreuth une relative autonomie artistique face aux institutions, le Reichskulturkammer de Goebbels et les instances du parti ${ }^{14}$. Prise entre les feux des traditionalistes wagnériens d'une part, les exigences antimodernes nazies d'autre part, elle suivit le conseil de son mari et imposa comme directeur artistique le metteur en scène et chef d'orchestre Heinz Tietjen, déjà directeur des théâtres prussiens à Berlin avant 1933. Ce dernier avait d'abord été proche de la gauche libérale avant de devenir le protégé de Göring et le responsable de mises en scène scandaleuses à la Krolloper de Berlin. Elle lui associa, toujours selon le vœu de Siegfried, Emil Preetorius, un décorateur reconnu, influencé modérément par Appia et par l'expressionnisme, et le fit épauler par le technicien d'éclairage Paul Eberhardt, promu alors directeur technique. De nouveaux chefs d'envergure internationale comme Toscanini dans les premiers temps, puis ensuite Furtwängler et Richard Strauss, ainsi qu'une nouvelle génération de chanteurs de qualité extraordinaire, favorisèrent ce nouveau départ.

Le renouveau consista non seulement à élever la qualité musicale au niveau de celle d'une capitale (Weltstadtniveau), mais surtout à " déblayer " la scène ancienne : Tietjen, qui se qualifiait lui-même ainsi que ses collaborateurs de " déblayeurs " (Entrümpler), appelait à une " création d'espace ", en affirmant que " l'image scénique, le décor s'étaient mus en espace scénique (Bühnenraum) ${ }^{15}$ » et Preetorius définissait sa tâche comme un " évidement de la scène (Bühne frei machen) " ${ }^{16}$. 


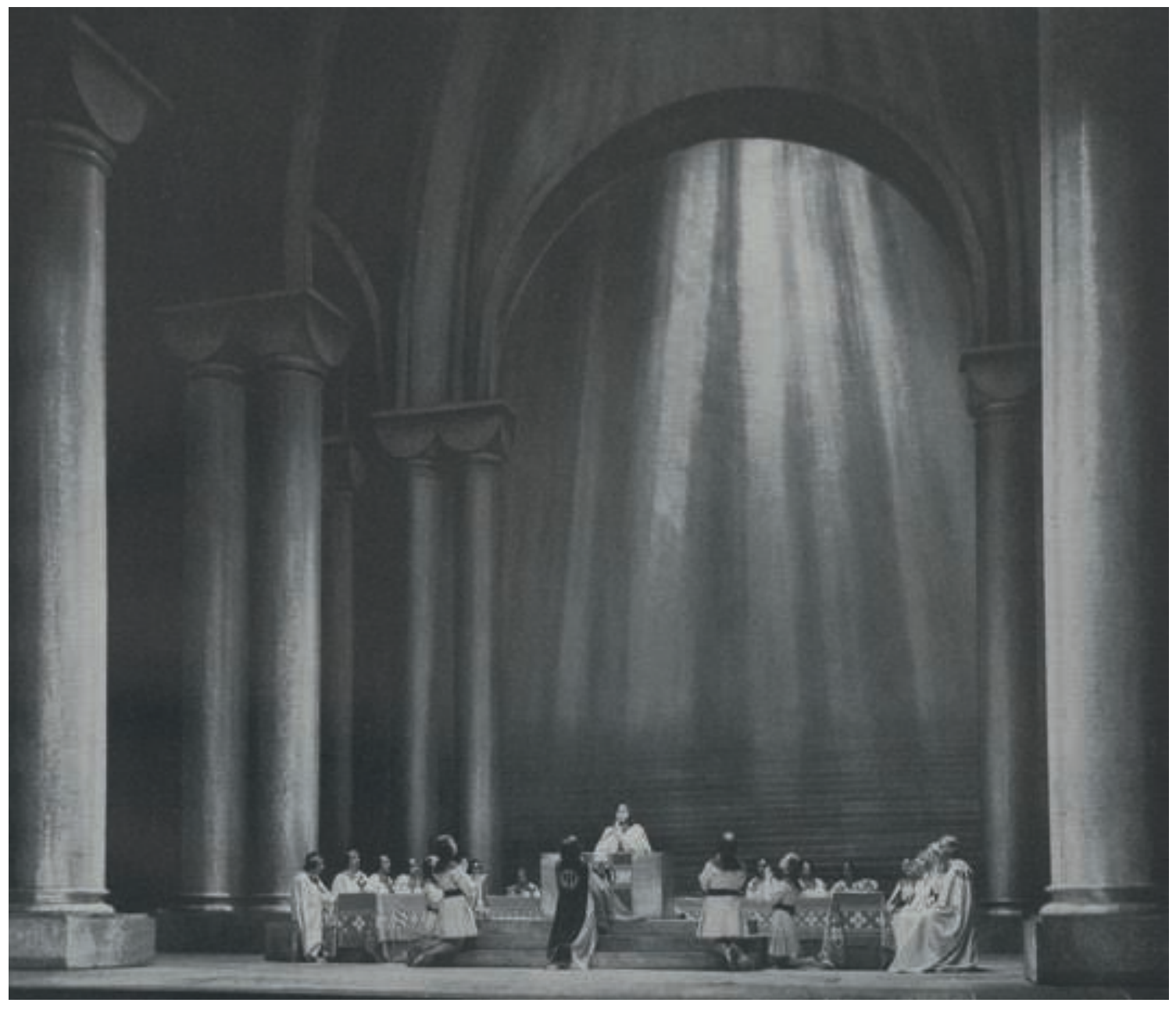

Décor d'Emil Preetorius pour Parsifal au Staatsoper de Hambourg en 1936, avec une mise en scène d'Oskar Fritz Schuh (in Emil Preetorius, Das szenische Werk, Berlin, Limbach, 1941 ; coll. BNU) 


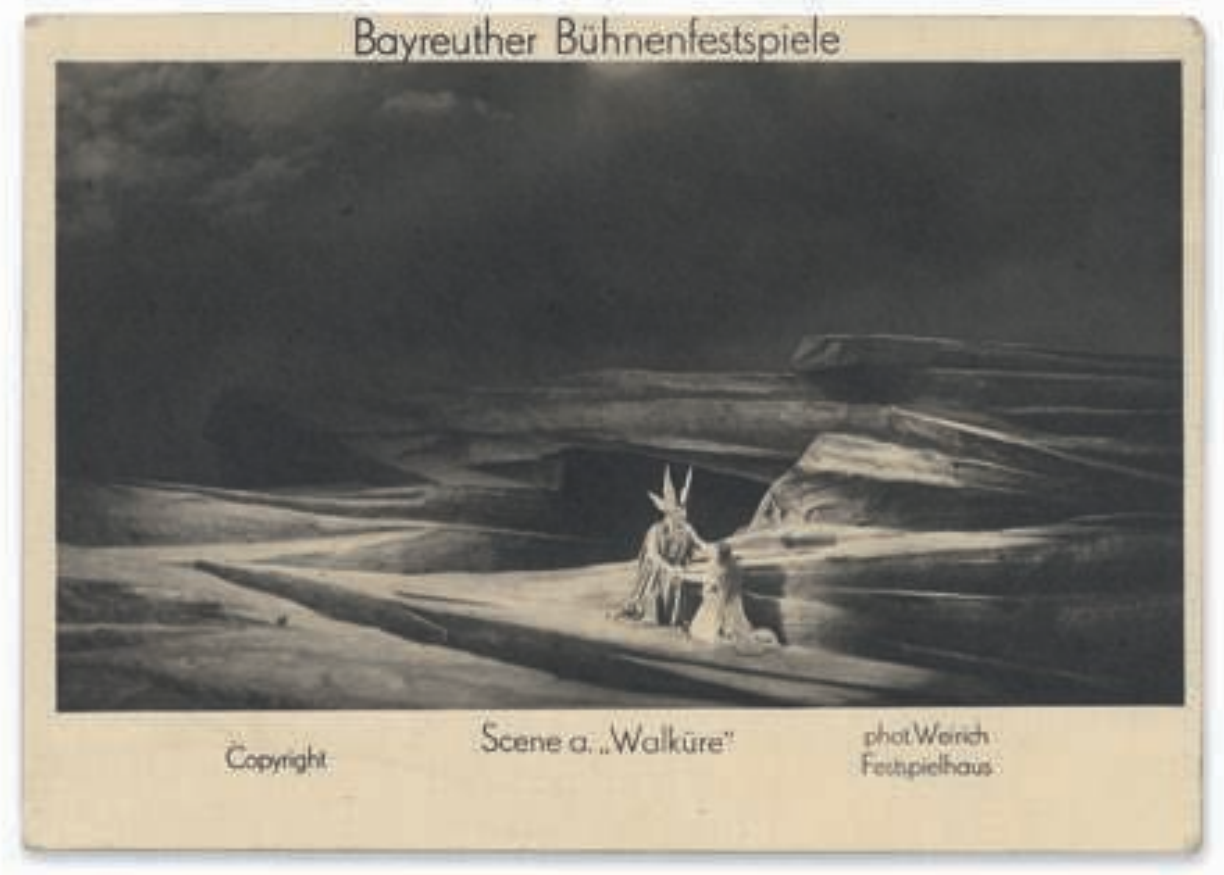

Décor de La Walkyrie par Emil Preetorius, carte postale éditée lors de la représentation du Festival de Bayreuth de 1937 (direction musicale W. Furtwängler, mise en scène H. Tietjen, décors E. Preetorius ; coll. BNU)

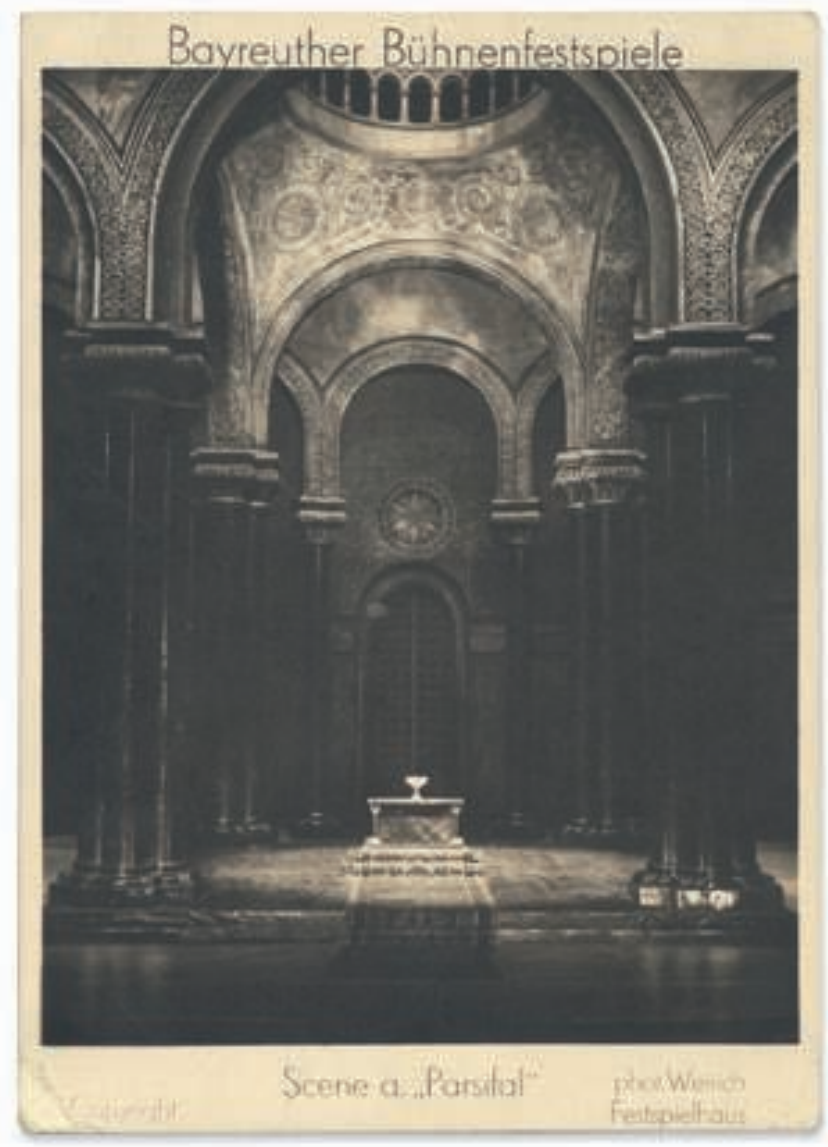

Décor de Parsifal par Wieland Wagner, carte postale éditée lors de la représentation du Festival de Bayreuth de 1937 (direction musicale W. Furtwängler, mise en scène H. Tietjen, décors W. Wagner ; coll. BNU) 
Ce dernier insistait en outre sur l'importance de l'installation des éclairages : "Wagner est une affaire de lumière ".

Les Maîtres chanteurs et les parties montrées de la Tétralogie en 1933 créèrent ainsi, avec une simplification du décor et des costumes, une " monumentalisation dela scène immense " (Frida Leidner) qui systématiquement marquait les ébauches de Siegfried Wagner au coin d'une esthétique rappelant à la fois la magie des lumières et la chorégraphie des masses de Max Reinhardt, et surtout l'esthétique cinématographique des Nibelungen de Fritz Lang : d'après les photos de scène du Ring ${ }^{17}$ par exemple, la scène, les costumes et les masques, jusqu'au Siegfried de Max Lorenz, y ont visiblement fait des emprunts.

Cette influence du cinéma, prégnante aussi dans la mise en scène de la politique nazie sous l'égide d'Albert Speer, pouvait médiatiser une modernité " modérée " encore acceptable pour le goût du Reich en art. Ce rattrapage avait son prix cependant : la nécessité de s'afficher comme enseigne artistique internationale du nouveau régime ${ }^{18}$. Cette modernité du début du siècle entra explicitement à Bayreuth en 1934 pour la nouvelle production de Parsifal, voulue et financée par Hitler. Son décorateur Alfred Roller avait été proposé par le Führer lui-même, qui admirait son art depuis qu'il avait vu en 1908 ses mises en scène à l'Opéra de Vienne ${ }^{19}$.

Ici s'impose une remarque concernant la wagnérophilie notoire du dictateur. Tandis que Bayreuth devenait, selon l'expression de Thomas Mann, une sorte de " théâtre de cour " pour Hitler qui s'y comportait en connaisseur, en esthète, et à ses heures en artiste parmi les artistes, Wagner fut consacré compositeur officiel pour les célébrations d'État comme le 21 mars 1933 lors de l'ouverture du Reichstag, à laquelle participa le Festspielchor de Bayreuth ${ }^{20}$, et qui fut suivie, le soir, d'une présentation des Maîtres chanteurs à la Staatsoper de Berlin sous la direction de Furtwängler ; ou comme lors de la réunion annuelle du parti à Nuremberg, inaugurée par l'ouverture de Rienzi et close avec Les Maîtres chanteurs par les soins de Benno von Arent, le « décora- teur du Reich ", qui en avait travaillé la scénographie avec le Führer lui-même. Wagner fut omniprésent dans le projet nazi d'une esthétisation de la politique ${ }^{21}$ qui mettait en scène une chorégraphie nocturne des masses sous un éclairage sophistiqué et théâtral. Wagner était pour Hitler non seulement un modèle dans l'art de séduire et d'exercer une emprise sensorielle sur les masses, mais aussi le penseur d'un Etat conçu comme œuvre d'art totale, dont le dictateur se considérait comme "l'artiste et le sauveur " ${ }^{22}$.

Une telle conception artistique avait d'abord façonné l'expérience scénographique d'Alfred Roller, le seul professeur qui voulut accepter, semble-t-il, l'aspirant artiste de Linz aux BeauxArts de Vienne. En 1903, Roller avait proposé, sous la direction de Gustav Mahler, un Tristan dont les décors plus sobres et stylisés étaient transformés par la lumière et ses changements de couleur en un espace correspondant aux affects suggérés par la musique. Pendant son séjour viennois de quelques mois, Hitler avait vu plusieurs fois toutes ses mises en scènes wagnériennes, dont Tristan et le Ring, qu'il aimera vanter plus tard, devant témoins, comme expériences décisives ${ }^{23}$.

Malgré la vieillesse et la maladie, Roller proposa sous la direction de Richard Strauss une conception scénique pour Parsifal qui créa un scandale et fit de la coupole de la salle du Graal l'enjeu d'une querelle opposant wagnériens traditionalistes et partisans d'une modernité début de siècle. L'entrée des chevaliers dans le temple du Graal en fut la pierre d'achoppement :

" La musique dure si longtemps que les chevaliers sont obligés dans la plupart des mises en scènes d'arpenter la scène plusieurs fois, ce qui ne paraissait pas sensé pour Roller. Il profitait donc pleinement de l'immense espace scénique du Festspielhaus, soulignait la profondeur du plateau par des colonnes dont la hauteur, dans un effet de perspective, diminuait de plus en plus vers le fond et qui prenaient l'apparence d'une forêt $"{ }^{24}$.

La coupole avait disparu et avec elle le référent au sacré. La rédemption devenait immanente - et renvoyait implicitement au " mécène " qui avait fait 
engager Roller. De cette manière, la modernité du projet pouvait satisfaire le commanditaire. Les traditionalistes wagnériens lui reprochaient par contre de représenter un " hall de congrès " ou "d'hôtel " 25 .

Deux ans plus tard, la coupole est restituée par la main du fils de Winifred et protégé d'Hitler, Wieland, qui signera aussi de nouveaux décors pour le Parsifal de $1937^{26}$. Renouant avec l'ancienne tradition et s'opposant explicitement à l'esthétique de l'équipe Tietjen/Preetorius, Wieland affirme de la sorte une première autonomie artistique. Elle est contrainte par le contexte d'un horizon fermé : l'alternative à l'esthétique de Tietjen et Preetorius consiste ou bien à revenir à la tradition wagnérienne ou bien à se montrer solidaire avec le goût antimoderne et provincial que défendent Goebbels et le parti. D'un côté Wieland flirte avec les ennemis de l'équipe qui dirige le Festspielhaus, Goebbels et le décorateur du Reich, Benno von Arent, auquel Winifred et Tietjen avaient barré l'accès à la scène de Bayreuth ; de l'autre, il cherche à s'y imposer comme homme de théâtre et futur directeur du festival. On a interprété cette opposition de Wieland au premier Bayreuth comme l'effet d'une lutte œdipienne contre Tietjen et sa mère, et comme la recherche d'une autonomie artistique chez ce jeune homme de vingt ans qui voulait être reconnu en tant que seul héritier légitime et en tant qu'artiste ${ }^{27}$. L'influence de l'esthétique nazie officielle, proche de la scénographie de Benno von Arent, s'affirmera avec ses propres esquisses pour Les Maîtres chanteurs de 1943/44 lors du "festival de la guerre ". Avec le retour d'un Nuremberg médiéval, il y renoue non seulement avec la tradition wagnérienne, mais crée aussi " les décors les plus favorables au régime de l'ère Winifred/Tietjen ${ }^{28}$.

\section{Le Neu-Bayreuth de 1951}

Tandis que le travail de Tietjen et Preetorius gagnait après la guerre les scènes nationales et internationales en tant que "pont entre les années 40 et 50 " 29 , les deux frères, Wieland et Wolfgang, qui prirent la direction de Bayreuth ${ }^{30}$, placèrent le festival sous le signe d'un art décidément apolitique qui devait débarrasser Bayreuth à la fois du fatras historique ancien et de l'idéologie du héros et du mythe germaniques: " Nous avons démonté le style Bayreuth. Nous ne nous intéressons plus aux dieux germaniques, mais à l'Homme ${ }^{31}$.
Jusqu'à sa mort en 1966, c'est l'art de Wieland qui fit rayonner ce programme par le recours systématique à la modernité d'Appia et à l'art abstrait. La mise en scène de Wieland réussit ainsi à détruire la fixation imaginaire du discours musical dans une iconographie illustrative dont les valeurs politiques et idéologiques étaient devenues obsolètes. Partant de la partition, une scénographie en mouvement, à la fois abstraite et sensuelle par ses couleurs, dialoguait avec la musique wagnérienne pour donner au spectateur la possibilité d'investir singulièrement l'espace entre l'œil et l'oreille. Ses mises en scène, ainsi doublées d'une scénographie rigoureuse, tendaient à faire vivre le drame psychique archaïque en chorégraphiant de façon stylisée les actions théâtrales. Cette chorégraphie était dirigée par des représentants de la danse allemande expressionniste (dont l'épouse de Wieland) et élaborée à partir des affects rendus audibles par la musique. Ainsi l'aspect visuel de la mise en scène et de la scénographie avait à la fois une fonction analytique et une fonction affective : les parois de l'espace scénique qui en arrière fermaient la scène en un demicercle de cyclorama, ainsi que les costumes et les rares objets scéniques, frappaient par la qualité sensible de leurs matériaux, et pouvaient ainsi susciter des investissements affectifs, telles les différentes textures de cuir pour les costumes, les matériaux souples, doux ou durs du décor, qui éveillaient le sens tactile du spectateur. La régie de l'éclairage rendait ces qualités sensuelles et créait une correspondance entre les impressions visuelles et auditives.

D'après les descriptions et les analyses de ces mises en scène qui mettaient en avant la régie de l'éclairage ${ }^{32}$ avec l'aide du "Lichtmeister " Paul Eberhardt, employé déjà par Tietjen, il apparaît que le but de Wieland Wagner était que le spectateur perçoive l'action scénique comme une " hallucination " se signifiant elle-même en tant que projection imaginaire. Ainsi le panneau de gaze qui fermait le cadre de l'ouverture de la scène éloignait les acteurs derrière un "voile de perception". La régie de l'éclairage brouillait les contours de l'espace et des objets en les faisant sans cesse osciller imperceptiblement dans des nuances de couleur subtiles. Des projections de configurations vagues, sur l'écran du cadre scénique et sur le cyclorama du fond de la scène, renforçaient l'impression hallucinatoire. Le cyclorama et une sphère ronde ou ovale légèrement penchée vers l'avant ${ }^{33}$, servant de " cothurne " au jeu selon les mots de Wieland, étaient aussi des symboles d'un univers archétypal dans lequel seul l'Homme, et plus encore le 


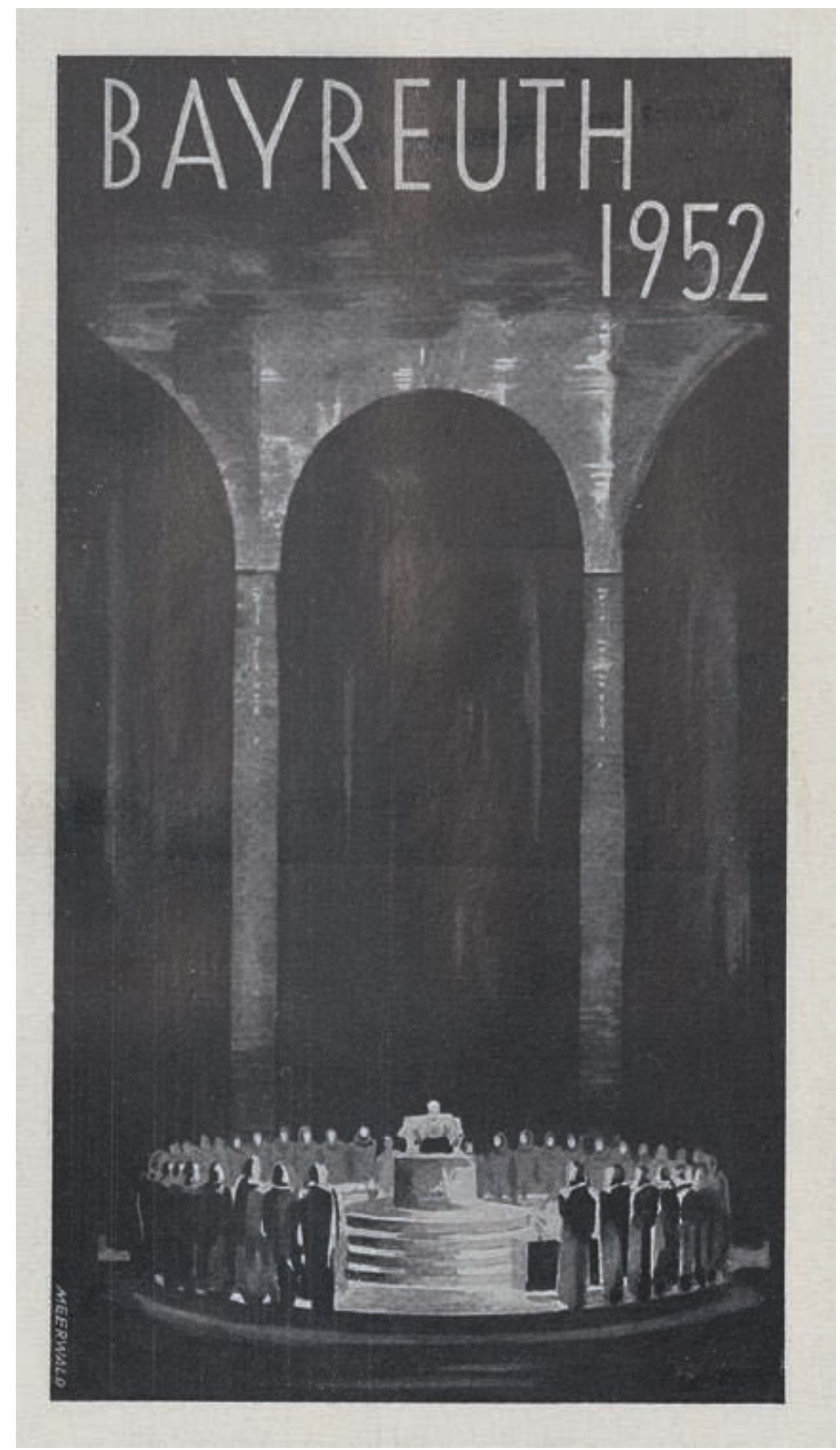

Page de programme du Festival de Bayreuth 1952 pour la représentation de Parsifal (direction musicale $\mathrm{H}$. Knappertsbusch, mise en scène et décors Wieland Wagner ; coll. BNU) 


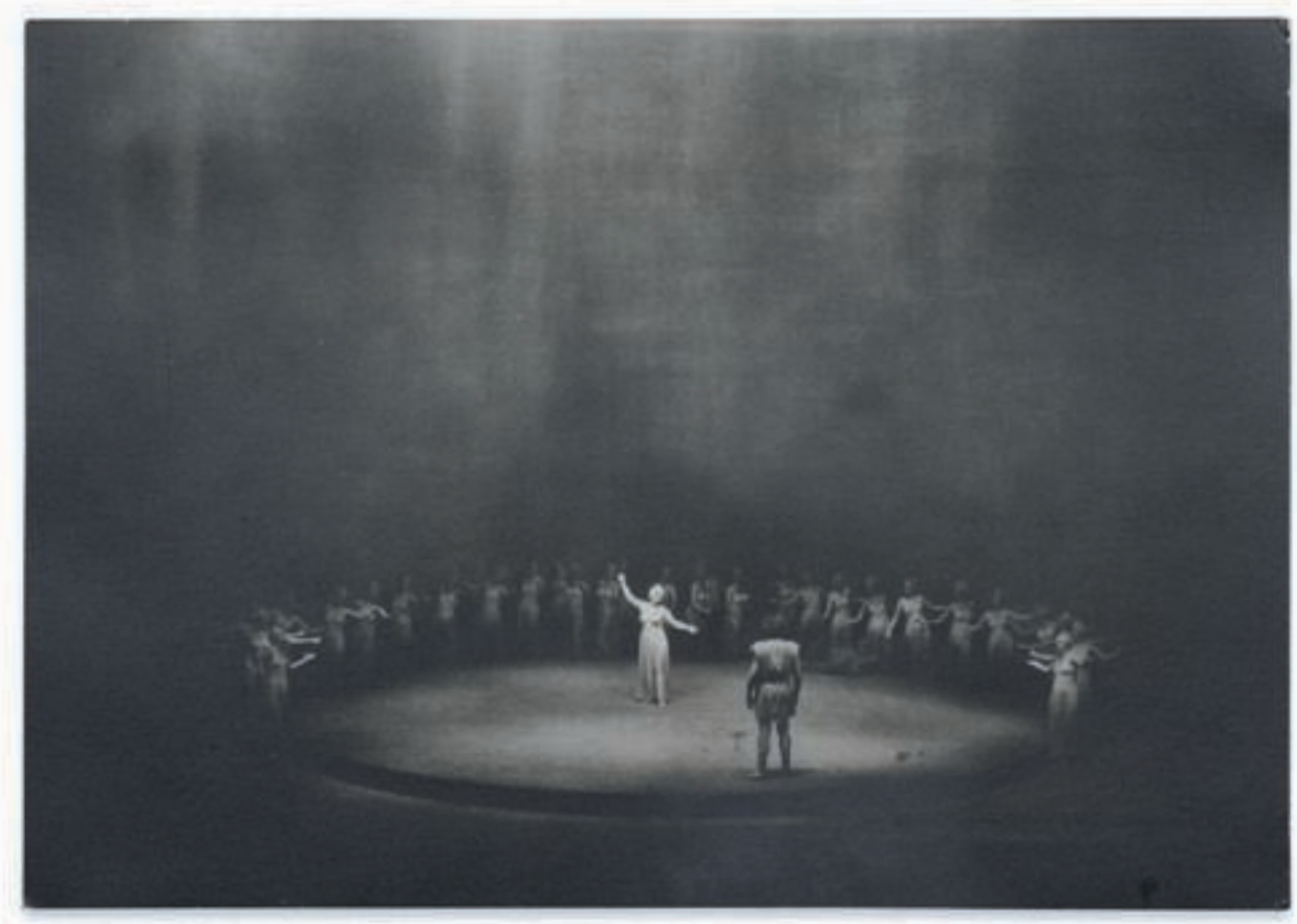

Décor pour l'acte 2 de Parsifal lors du Festival de Bayreuth 1952

(direction musicale H. Knappertsbusch, mise en scène et décors Wieland Wagner ; coll. BNU)

Héros, surgissait éclairé. Wieland explorait, à partir du drame préfiguré dans la partition, le drame psychique que la musique faisait entendre et pour lequel il chercha des codes visuels, inspirés à partir de 1965 de l'art brut et de la sculpture de son époque ${ }^{34}$.

Si l'on en croit les récits des contemporains, on peut affirmer que Wieland Wagner avait réussi à transformer le théâtre de son grand-père en un "Lichtspiel ", un jeu de lumières, convoquant une sorte de cinéma sublime inversé, car c'était maintenant la musique qui motivait l'image. Son style évolua avec le temps : « Du vide, des ténèbres et de la ligne pure des premières mises en scène, il parvint à une plasticité différenciée, proposa des symboles iconographiques et des effets de lumières stylisés ", note Nike Wagner qui interprète cette évolution comme métaphore de la recherche d'un sujet, enfin débarrassé de son moi ancien aliéné par le contexte familial et historique ${ }^{35}$.

Enveloppé d'un espace sonore dont le demi-cercle, en rejoignant celui de l'arrière-scène, se fermait en cercle complet, le spectateur pouvait vivre l'action scénique comme une projection fantasmatique générée par le son, aussi bien sur le mode sensoriel d'une unité audiovisuelle que sur un mode analytique et distancié. Car les écrans de cette projection étaient signalés en tant que tels, et la chorégraphie des mouvements des chanteurs empêchait par sa logique propre l'attente d'une sym- biose entre affect audiovisuel et imaginaire. Ainsi, l'art de Wieland situait le drame wagnérien dans un classicisme atemporel, remplaçant le référent germanique par un autre, grec ou plus tard archétypal ou shakespearien, comme par exemple dans les derniers Maîtres chanteurs.

Cette esthétique analytique, fondée sur l'abstraction et la symbolisation, connut un rayonnement en dehors de Bayreuth, et des éléments en furent imités çà et là. Mais l'esprit de ce style ne trouva pas de successeur immédiat à Bayreuth. Une nouvelle historicisation se fit jour, qui réactualisait l'œuvre de Wagner et la réinterprétait à partir du contexte politico-social de sa production. La fusion de la vision (psycho-) analytique de Wieland avec ce courant se réalisera dans le Ring de Patrice Chéreau et de Pierre Boulez, en 1976.

Wolfgang Wagner ouvrit ensuite Bayreuth à de nouvelles expériences, dont le Tristan de Heiner Müller, Erich Wonder et Daniel Barenboim de 1993-95, le Parsifal de Christoph Schlingensief de 2004-2007, le Lohengrin d'Hans Neuenfels de 2010-2012, et enfin le Parsifal de Stefan Herheim de 2008-2012 sont les représentants les plus marquants. D’autres expériences innovatrices se firent ailleurs, comme celle de Ruth Berghaus, Axel Manthey et Michael Gielen à Francfort dans les années 80 , celle de Robert Wilson à Hambourg et à Zurich, ou celle de Klaus Michael Grüber à Salzbourg et Bruxelles à partir des années go. 
Plusieurs tendances se dessinent aujourd'hui pour l'avenir du "Gesamtkunstwerk " : celle qui allie spatialement l'œil et l'oreille, mettant en avant la musique et la lumière tout en comprenant la musique comme génératrice d'images scéniques comme chez Wieland, Wilson, Müller ou Grüber. Le spectateur est ainsi invité à analyser son propre désir de voir et d'entendre et à jouir d'un plaisir à la fois sensuel et intellectuel. Puis la tendance qui sature l'œil en lutte avec la musique, comme au premier acte du Parsifal de Schlingensief. Enfin, celle qui dirige l'écoute par une mise en scène dont le visuel déconstruit l'action, analyse le mythe, critique la politique de notre époque comme celle qui était contemporaine à la production de l'œuvre - sans oublier les mises en scène illustratives qui perdurent et plaisent toujours, parce qu'elles proposent une scénographie en adéquation avec une rhétorique théâtrale reconnue et avec un référent artistique familier, fût-il celui des arts à la mode.

Par goût esthétique, par inclination personnelle, je préfère la première tendance dans le sillon du travail de Wieland. Mais l'exemple d'Herheim, cité au début de cette réflexion, me paraît une alternative probante, car il brasse l'imaginaire de la scénographie wagnérienne et du wagnérisme d'une telle façon que, malgré le foisonnement des objets, le dédoublement des personnages, le spectateur dans son écoute n'est pas dirigé idéologiquement par le propos visuel. Car les créations visuelles changent continuellement comme dans un kaléidoscope, ce qui incite le spectateur à concevoir lui-même sans cesse son propre espace imaginaire et à le mettre en rapport avec la musique, ce qui lui est aisément possible parce que tout ce qui est montré sur scène est motivé et justifié à partir de la partition. Ces deux options de mise en scène, enfin, réussissent à relever le défi de réaliser le projet du " Gesamtkunstwerk ", celui d'une potentielle synthèse des sens, tout en permettant de la construire par l'intelligence.

\section{Helga Finter}

\section{Notes}

1 - Richard Wagner, Parsifal : ein Bühnenweih-Festspiel, Stuttgart, Reclam, 1962, p. 24 et suiv.

2 - Pour une description détaillée et une riche documentation iconographique, cf. Antonia Goldhammer, Weißt du, was du sahst? Stefan Herheims Bayreuther Parsifal, Berlin, München, Deutscher Kunstverlag, 2011.

3 - Ibid., p. 11-12

4 - Si déjà avec le Ring de Chéreau et de Boulez en 1976, qui thématisait le contexte historique de la création de l'œuvre, la politique était rentrée sur la scène de Bayreuth, une interprétation scénique des implications avec l'esthétique du Troisième Reich était un fait nouveau.
5 - Cf. Ingrid Kapsamer, Wieland Wagner. Wegbereiter und Weltwirkung, Graz, Styria Premium, 2010, p. 41

6 - Ibid., p. 102

7 - Cf. Richard Wagner, Die Kunst und die Revolution [1849], Das Kunstwerk der Zukunft [1849], in Richard Wagner, Ausgewählte Schriften, Frankfurt-am-Main, Insel, 1975 ; Richard Wagner, Oper und Drama [1851], Stuttgart, Reclam, 1984

8 - Cf. Richard Wagner, Das Bühnenfestspiel zu Bayreuth [1873], in Gesammelte Schriften und Dichtungen, t. 9, Leipzig, 1883, p. 384 et suiv.

9 - Cosima Wagner, Journal III, 1878-188o, texte établi et commenté par Martin Gregor-Dellin et Dietrich Mack, traduit par Michel-François Demet, Paris, Gallimard, 1977, p. 194

10- Oswald G. Bauer, Die Aufführungsgeschichte in Grundzügen, in Ulrich Müller et Peter Wapnewski, Richard-Wagner-Handbuch, Stuttgart, A. Kröner, 1986, p. 647-674; ibid., p. 649, 652

11 - Matthias Nöther, Als Bürger leben, als Halbgott sprechen. Melodram, Deklamation und Sprechgesang im wilhelminischen Reich, Köln, Weimar, Wien, Böhlau, 2008, p. 114-128

12 - Oswald G. Bauer, op. cit., p. 653

13 - Cf. Philippe Olivier, Der Ring des Nibelungen in Bayreuth von den Anfängen bis heute, Mainz, Schott, 2007, p. 67-84

14 - Cf. la somme de Brigitte Hamann, Winifred Wagner oder Hitlers Bayreuth, München, Piper, 2003

15- Cités ibid., p. 225

16 - Ibid., p. 224

17 - Cf. Philippe Olivier, op. cit., p. 85-100

18 - Un autre prix à payer fut l'interdiction d'engager des artistes juifs à partir d'avril 1933 ; voir à ce sujet Verstummte Stimmen. Die Bayreuther Festspiele und die "Juden " 1876-1945, eine Ausstellung von Hannes Heer, Jürgen Kesting, Peter Schmidt, Berlin, Metropol, 2012.

19-Cf. Brigitte Hamann, Hitlers Wien. Lehrjahre eines Diktators, München, Piper, 1998, p. 87-95

20-Hamann 2003, p. 286

21 - Cf. Philippe Lacoue-Labarthe, La fiction du politique. Heidegger, l'art et la politique, Paris, Christian Bourgois, 1987, p. 92-113

22-Cf. Éric Michaud, Un art de l'éternité. L'image et le temps du nationalsocialisme, Paris, Gallimard, 1996, p. 15-123; pour la relation entre voix du dictateur et musique wagnérienne, cf. Michel Poizat, Vox populi, vox Dei. Voix et pouvoir, Paris, Métailié, 2001, p. 151-228

23 - Cf. Hamann, 1998, op. cit. Des esquisses de décor de la main d'Hitler pour Tristan (cf. Michaud, p. 85) ou pour Lohengrin (cf. Hamann 1998, p. 96) témoignent de cette influence.

24-Hamann 2003, p. 284

25 - Ibid., p. 185

26 - Cf. la reproduction d'une esquisse de Wieland, in I. Kapsamer, op. cit., p. 102

27 - Cf. Hamann 2003, p. 486-49o ; I. Kapsamer, p. 33-79 ; Nike Wagner, Wagner Theater, Frankfurt-am-Main, Suhrkamp, 1999, p. 307-319

28-Hamann 2003, p. 465

29-Oswald G. Bauer, op. cit., p. 669 et suiv.

30 - Les deux frères avaient joui d'une formation de metteur en scène depuis la fin des années trente, Wolfgang par Tietjen, Wieland de sa propre initiative, menant chez ce dernier à une mise en scène du Ring en 1943/44, donné simultanément à Nuremberg et à Altenburg (cf. Nike Wagner, op. cit., p. 312 ; Kapsamer, op. cit., p. 79-87)

31 - Wolfgang et Wieland Wagner à la conférence de presse de clôture de la première saison après la guerre, en 1951, cités par Wolfgang Greisenegger, in Kapsamer, op. cit., p. 12.

32 - Cf. Claude Lust, Wieland Wagner et la survie du théâtre lyrique, Lausanne, 1969 ; Ingrid Kapsamer, Wieland Wagner. Wegbereiter und Weltwirkung, Graz, Styria, 2010.

33 - Elle fut introduite après son invention par Wolfgang dans son Ring de 1960, cf. Philippe Olivier, p. 123 et suiv.

34 -Cf. ibid., p. 121 et suiv.

35-Cf. Nike Wagner, op. cit., p. 238 